\title{
Spectroscopic Evidence for an Additional Symmetry Breaking in the Nematic State of FeSe Superconductor
}

Cong Li, ${ }^{1,2}$ Xianxin Wu, ${ }_{1,3}^{1,3}$ We Wang, ${ }^{1,2}$ Defa Liu, ${ }^{1,4}$ Yongqing Cai, ${ }^{1,2}$ Yang Wang, ${ }^{1,2}$ Qiang Gao, ${ }^{1,2}$ Chunyao Song, ${ }^{1,2}$ Jianwei Huang, ${ }^{1,2}$ Chenxiao Dong, ${ }^{1,2}$ Jing Liu, ${ }^{1,2}$ Ping Ai, ${ }^{1,2}$ Hailan Luo, ${ }^{1,2}$ ChaoHui Yin,${ }^{1,2}$ Guodong Liu, ${ }^{1}$ Yuan Huang, ${ }^{1}$ Qingyan Wang, ${ }_{1}$ Xiaowen Jia, ${ }^{5}$ Fengfeng Zhang, ${ }^{6}$ Shenjin Zhang, ${ }^{6}$ Feng Yang, ${ }^{6}$ Zhimin Wang, ${ }^{6}$ Qinjun Peng, ${ }^{6}$ Zuyan Xu, Youguo Shi, ${ }^{1,2}$ Jiangping Hu, ${ }^{1,2,9}$ Tao Xiang, ${ }^{1,2,9}$ Lin Zhao, ${ }^{1, *}$ and X. J. Zhou ${ }^{1,2,7,8, \uparrow}$

${ }^{1}$ Beijing National Laboratory for Condensed Matter Physics, Institute of Physics, Chinese Academy of Sciences, Beijing 100190, China

${ }^{2}$ University of Chinese Academy of Sciences, Beijing 100049, China

${ }^{3}$ Institute for Theoretical Physics and Astrophysics, Julius-Maximilians University of Wurzburg, Am Hubland, D-97074 Wurzburg, Germany

${ }^{4}$ Max Planck Institute of Microstructure Physics, Weinberg 2, Halle 06120, Germany

${ }^{5}$ Military Transportation University, Tianjin 300161, China

${ }^{6}$ Technical Institute of Physics and Chemistry, Chinese Academy of Sciences, Beijing 100190, China

${ }^{7}$ Songshan Lake Materials Laboratory, Dongguan 523808, China

${ }^{8}$ Beijing Academy of Quantum Information Sciences, Beijing 100193, China

${ }^{9}$ Collaborative Innovation Center of Quantum Matter, Beijing 100190, China

(Received 13 February 2020; revised 11 May 2020; accepted 16 June 2020; published 12 August 2020)

\begin{abstract}
The iron-based superconductor FeSe has attracted much recent attention because of its simple crystal structure, distinct electronic structure, and rich physics exhibited by itself and its derivatives. Determination of its intrinsic electronic structure is crucial to understanding its physical properties and superconductivity mechanism. Both theoretical and experimental studies so far have provided a picture that FeSe consists of one holelike Fermi surface around the Brillouin zone center in its nematic state. Here we report direct observation of two holelike Fermi surface sheets around the Brillouin zone center, and the splitting of the associated bands, in the nematic state of FeSe by taking high-resolution laser-based angle-resolved photoemission measurements. These results indicate that, in addition to nematic order and spin-orbit coupling, there is an additional order in FeSe that breaks either inversion or time-reversal symmetries. The new Fermi surface topology asks for reexamination of the existing theoretical and experimental understanding of FeSe and stimulates further efforts to identify the origin of the hidden order in its nematic state.
\end{abstract}

DOI: 10.1103/PhysRevX.10.031033

Subject Areas: Strongly Correlated Materials, Superconductivity

In iron-based superconductors, FeSe has the simplest crystal structure which consists solely of the basic building block, the FeSe layers, that dictates the superconductivity [1]. It undergoes a structural phase transition at around $90 \mathrm{~K}$ to enter a nematic state but without a long-range magnetic order [2]. Superconductivity of FeSe can be significantly enhanced under high pressure $[3,4]$. It represents a unique system that

\footnotetext{
* Corresponding author.

1zhao@iphy.ac.cn

Corresponding author. XJZhou@iphy.ac.cn

Published by the American Physical Society under the terms of the Creative Commons Attribution 4.0 International license. Further distribution of this work must maintain attribution to the author(s) and the published article's title, journal citation, and DOI.
}

superconductivity and nematicity coexist in the superconducting state, giving rise to its distinct electronic structure and superconducting gap structure [5-24]. Many new superconductors have been derived from FeSe with enhanced superconductivity, including $A_{x} \mathrm{Fe}_{2-y} \mathrm{Se}_{2}(A=\mathrm{K}, \mathrm{Rb}, \mathrm{Tl}$, etc.) $[25,26]$ and other organic intercalated superconductors [27-31], ( $\mathrm{Li}, \mathrm{Fe}) \mathrm{OHFeSe}$ [32], heavily electron-doped FeSe through gating or potassium deposition [33-35], and in particular, single-layer $\mathrm{FeSe} / \mathrm{SrTiO}_{3}$ films with a record high $T_{c}$ [36-40]. Determination of the electronic structure is essential to understanding the physical properties and superconductivity mechanism in bulk FeSe and its many derivatives. So far, all the angle-resolved photoemission (ARPES) measurements report only one holelike Fermi surface around the Brillouin zone (BZ) center in its nematic state [5-19]. Scanning tunneling microscope (STM) results [20-22] and quantum oscillation measurements $[10,23,24]$ are also 

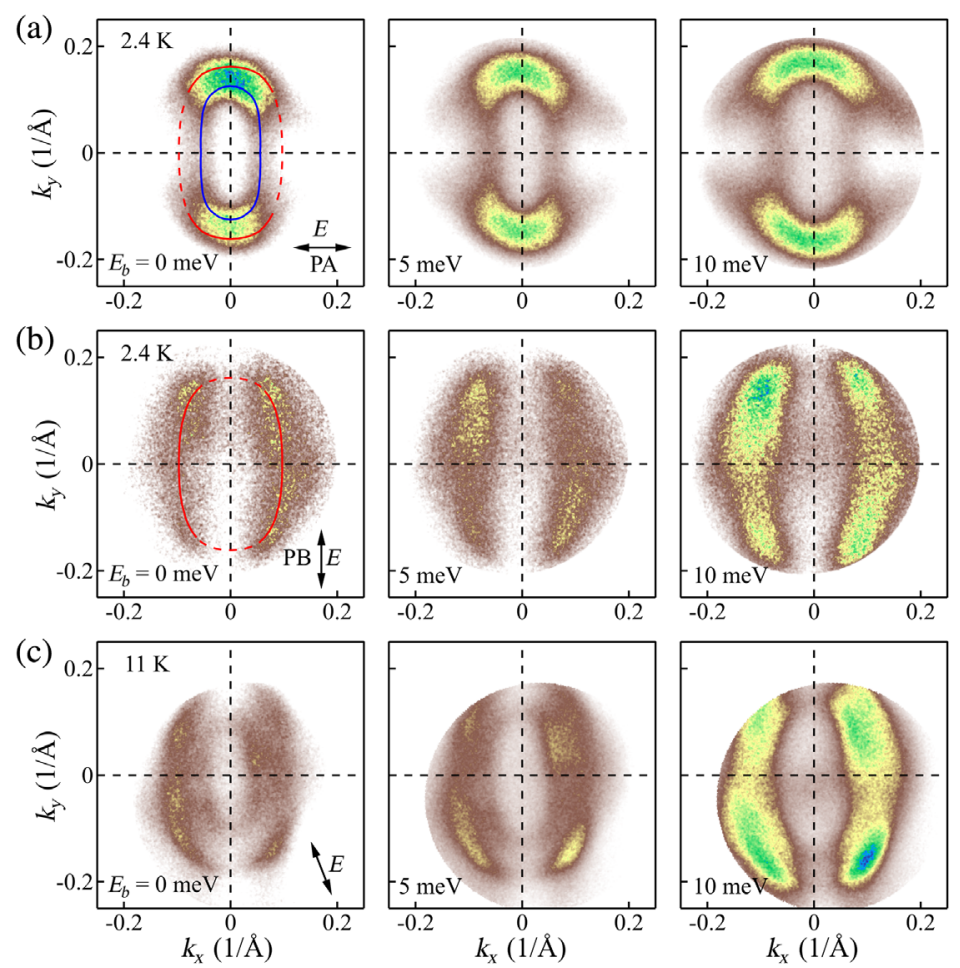

(d)

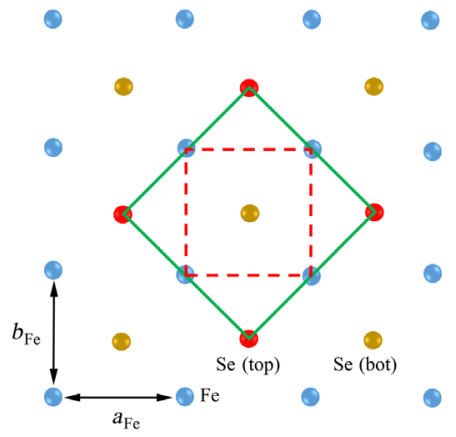

(e)

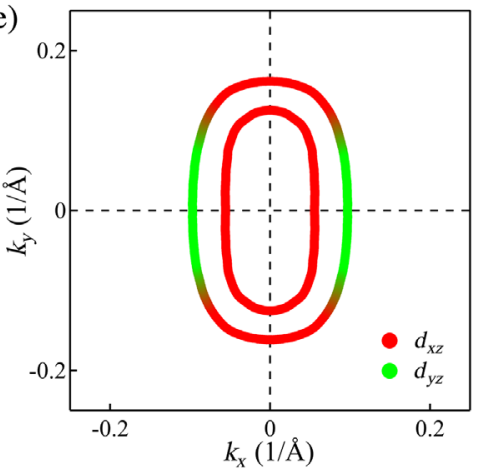

FIG. 1. Observation of two holelike Fermi surface sheets in single-domain FeSe. (a)-(c) Constant energy contours of the singledomain FeSe at $2.4 \mathrm{~K}$ under the PA polarization (a), at $2.4 \mathrm{~K}$ under the PB polarization (b), and at $11 \mathrm{~K}$ under a mixed polarization (c). The direction of the electric field vector $E$ corresponding to the three polarization geometries is marked by double arrows in the leftmost panels in (a)-(c). Blue and red ellipses in the leftmost panels are the guide lines of the observed Fermi surface. (d) Top view of FeSe crystal structure. The blue, red, and yellow circles represent Fe atom, Se atom above the Fe plane (Se top), and Se atom below the plane (Se bottom), respectively. The red dashed line represents the 1-Fe unit cell while the green solid line represents 2-Fe unit cell. We define the $x$ axis parallel to the $a_{\mathrm{Fe}}$ axis and the $y$ axis parallel to the $b_{\mathrm{Fe}}$ axis. In the normal state, FeSe has $C_{4}$ symmetry with $a_{\mathrm{Fe}}=b_{\mathrm{Fe}}$, but it breaks into $C_{2}$ symmetry in the nematic state with $a_{\mathrm{Fe}}>b_{\mathrm{Fe}}$. (e) The extracted Fermi surface of FeSe which consists of two holelike Fermi surface sheets around the BZ center. The dominant orbital character of the two Fermi surfaces is also marked.

understood by assuming only one holelike Fermi pocket in the nematic state of FeSe. The picture of one holelike Fermi surface in FeSe is theoretically understood by considering the nematicity and / or spin-orbit coupling [15,21,41,42]. Understanding of the physical properties [43-45] and the pairing mechanism of FeSe $[46,47]$ have been based on one Fermi pocket picture around the BZ center in the nematic state.

In this paper, we performed high-resolution laser-based ARPES measurements on the electronic structure of FeSe in the nematic state. Double holelike Fermi surface sheets around the Brillouin zone center, and the splitting of the associated bands, are observed for the first time in FeSe. These two Fermi surface sheets exhibit different orbital characters. These results indicate that there is an additional order in $\mathrm{FeSe}$, in addition to the nematic order and spinorbit coupling, that further breaks inversion or time-reversal symmetries to give rise to the band splitting. The new Fermi surface picture of FeSe asks for reexamination of the previous theoretical and experimental understandings and stimulates further efforts to identify the origin of the hidden order in its nematic state.
The ARPES measurements were performed by using our latest-generation laser-based system; the present observations were made possible because of its unique capabilities of simultaneous coverage of two-dimensional momentum, versatile laser light polarizations, and ultrahigh energy and momentum resolutions (see Methods below) [48]. Figure 1 shows the Fermi surface and constant energy contours of FeSe measured at low temperatures in its nematic state under different polarization geometries [Figs. 1(a)-1(c)]. The crystal structure of FeSe transforms from tetragonal to orthorhombic below $90 \mathrm{~K}$; the distance between adjacent $\mathrm{Fe}$ atoms becomes slightly different and $a_{\mathrm{Fe}}\left(b_{\mathrm{Fe}}\right)$ is defined as the long $x$ (short $y$ ) axis in Fig. 1(d). Although we did not detwin our FeSe samples intentionally, we find that, in some cases, we can measure on FeSe samples or areas which are dominated by a single domain. This may be caused by local strain that is present or exerted during the sample preparation process, and a similar phenomenon was also observed before [14,15]. Figures 1(a)-1(c) show results obtained from single-domain $\mathrm{FeSe}$. When the electric field vector of the incident laser light $E$ is aligned parallel to the $a$ axis (called PA polarization), the measured 
Fermi surface mapping [leftmost panel in Fig. 1(a)] shows an ellipsoidlike shape with the strong spectral weight mainly concentrated on the two vertex areas along the long axis ( $b$ direction) and much weaker spectral weight along the short axis ( $a$ direction). Upon increasing the binding energy, the corresponding constant energy contours increase in the overall area, consistent with the holelike nature of the observed Fermi surface. When the electric field vector of the laser light is switched to be parallel to the $b$ direction (called PB polarization), the Fermi surface mapping in Fig. 1(b) is dramatically different from that in Fig. 1(a). The spectral weight near the two vertex areas along the long axis is strongly suppressed. More remarkably, the observed Fermi surface size along the $a$ axis is obviously larger than that observed in Fig. 1(a). By setting the electric field vector of the laser light away from perfect $b$ axis and making use of the combined matrix element effects in Figs. 1(a) and 1(b), we can directly observe two holelike Fermi surface sheets in Fig. 1(c), which consists of the inner small hole pocket observed in Fig. 1(a) and outer large hole pocket observed in Fig. 1(b). Detailed analyses of the Fermi surface and related band structures below and in Fig. S1 (Supplemental Material [49]) provide further clear evidence on the double holelike Fermi surface sheets around the Brillouin zone, as depicted in Fig. 1(e). The Fermi surface mappings in the superconducting state are quite similar to those in the normal state (see Fig. S2 in Supplemental Material [49]), indicating that the Fermi surface splitting around $\Gamma$ is not caused by the superconducting transition at $T_{c}$.

The above results in Fig. 1 indicate that our clear observation of the two coexisting Fermi surface sheets around the zone center in single-domain FeSe comes from the measurement that utilizes the mixed light polarization [Fig. 1(c)]. It is also clear that pure polarization geometries alone, either PA in Fig. 1(a) or PB in Fig. 1(b), are not desirable in revealing the double hole pockets because the measured Fermi surface [Figs. 1(a) and 1(b)] is dominated by one sheet although some subtle signatures are present. This also explains why the double hole pockets are not identified in all the previous ARPES measurements [5-19], because in most cases pure polarization geometries like PA [Fig. 1(a)] or PB [Fig. 1(b)] were used. These observations can be understood by the particular orbital character of the two hole pockets and the associated photoemission matrix element effects. In the PA polarization geometry [Fig. 1(a)], for both the horizontal and vertical cuts, only the $d_{x z}$ orbital is allowed [14-16]. On the other hand, in the PB polarization geometry [Fig. 1(b)], the $d_{y z}$ orbital is allowed for both the momentum cuts. Therefore, from the spectral weight distribution along the two Fermi surface sheets measured in the PA [leftmost panel in Fig. 1(a)] and PB [leftmost panel in Fig. 1(b)] polarization geometries, the dominant orbital character along the two Fermi surface sheets can be determined, as shown in Fig. 1(e). The entire inner Fermi surface is dominated by the $d_{x z}$ orbital. For the outer Fermi surface sheet, the vertex area along the long axis is dominated by the $d_{x z}$ orbital while the area along the short axis is composed predominantly of the $d_{y z}$ orbital. Therefore, in the PA polarization geometry [Fig. 1(a)], the inner Fermi pocket is visible, but most of the outer Fermi pocket is suppressed except for the vertex areas along the long axis. This makes the measured Fermi surface dominated by seemingly one pocket but with strong and broad spectral weight near the vertex region along the long axis due to the coexisting inner and outer pockets [Fig. 1(a)]. In the PB polarization geometry [Fig. 1(b)], the entire inner Fermi pocket and the vertex areas along the long axis of the outer pocket are suppressed, leaving the measured Fermi surface dominated by one outer pocket with suppressed vertex areas along the long axis.

The observation of two Fermi surfaces around the zone center in single-domain FeSe provides a unified picture to understand the Fermi surface topology measured in the twinned samples. Figure 2 shows the Fermi surface mapping of a twinned FeSe sample measured in PA [Fig. 2(d)], PB [Fig. 2(e)], and a mixed [Fig. 2(f)] polarization geometries. For a direct comparison, we also replot the Fermi surface of single-domain FeSe in Figs. 2(a)-2(c) measured under the same polarization geometries. For photoemission matrix element analysis, the polarization geometry is schematically shown in Fig. 2(m) and the two kinds of domains are shown in Fig. 2(n). Here the axes in the lab coordinates are defined as $X, Y$, and $Z$ while the axes $x$ and $y$ are associated with the particular singledomain FeSe. Under a given polarization geometry, the Fermi surface mappings of the twinned sample [Figs. 2(d)2(f)] contain extra features that are not present in the singledomain sample [Figs. 2(a)-2(c)]. This can be well understood as the overlap of signals from two orthogonal domains. In the twinned FeSe sample, there are two types of domains, named as vertical domain and horizontal domain, that are orthogonal to each other [Fig. 2(n)]. From the single-domain data in Figs. 2(a)-2(c), by considering the two orthogonal domains, we can simulate results for the twinned sample as shown in Figs. 2(g)-2(i). For example, under the PA polarization geometry, Fig. 2(g) is obtained by summing up the signal of the vertical domain in Fig. 2(a) and that of the horizontal domain in Fig. 2(b) but rotated by $90^{\circ}$. The simulated results [Figs. 2(g)-2(i)] can well reproduce the measured results [Figs. 2(d)-2(f)]. In this case, the Fermi surface mappings of the twinned sample measured in different polarization geometries [Figs. 2(d)-2(f)] can be well understood from summation of signals from two types of orthogonal domains, as schematically shown in Figs. 2(j)-2(1). The simultaneous observation of four Fermi surface sheets in the twinned sample [Fig. 2(f)] provides further unambiguous evidence on the double Fermi surface around the zone center in the single-domain FeSe [Fig. 2(c)]. 

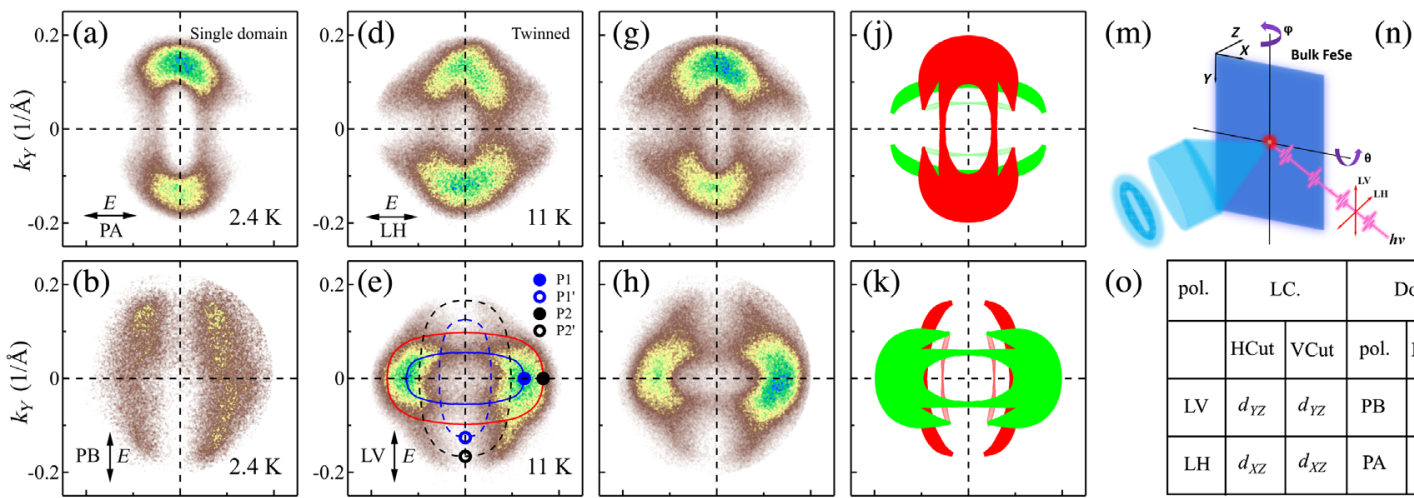

(n) $Y$

(o)
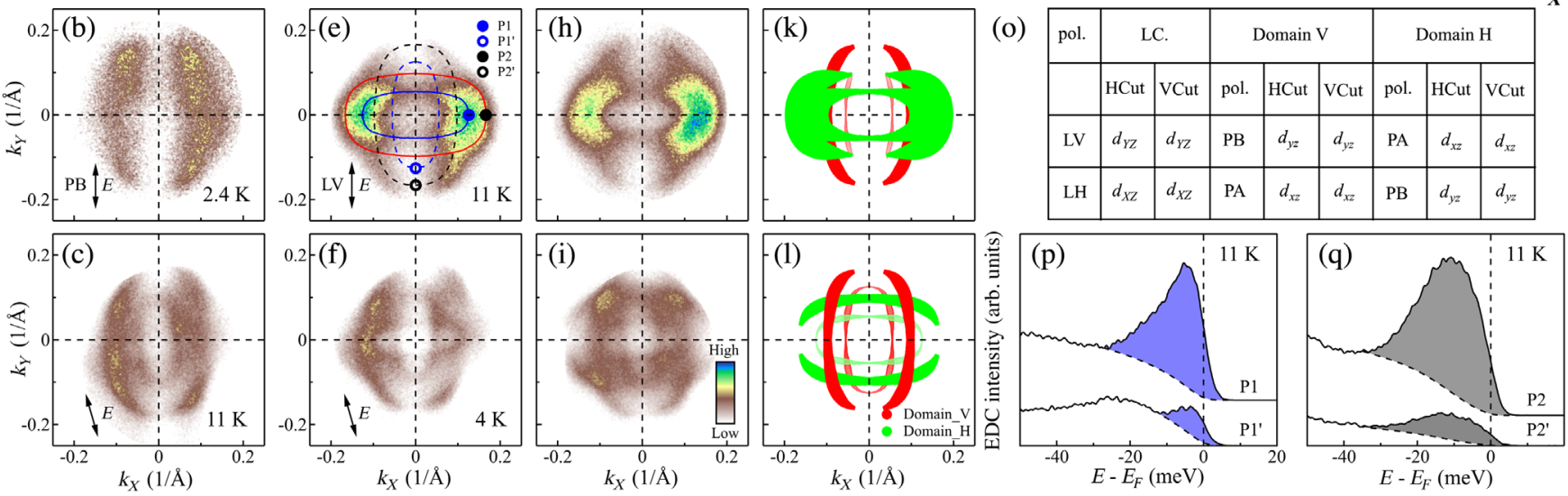

FIG. 2. Fermi surface mapping of twinned FeSe. (a)-(c) The Fermi surface mappings of the single-domain FeSe measured under the PA (a), the PB (b), and a mixed (c) polarization geometries. (d)-(f) The Fermi surface mappings of the twinned FeSe measured under the LH (d), the LV (e), and a mixed (f) polarization geometries. The measurement temperatures are marked at the bottom left corner in (a)-(f). (g)-(i) Simulated Fermi surface from the single-domain data in (a)-(c). Under the PA polarization geometry, (g) is obtained by summing up the signal of the vertical domain in (a) and that of the horizontal domain in (b) but rotated by $90^{\circ}$. Under the PB polarization geometry, (h) is obtained by rotating the data in $(\mathrm{g})$ by $90^{\circ}$. Under the mixed polarization geometry, (i) is obtained by the summation of signals from the vertical domain in (c) and from the horizontal domain also in (c) but rotated $90^{\circ}$. (j)-(l) Schematic of the observed Fermi surface in twinned FeSe from the horizontal domain (green lines) and the vertical domain (red lines) corresponding to data in (d)-(f). (m) Schematic drawing of the experimental geometry with LV and LH polarizations. $X, Y$, and $Z$ axes are defined as laboratory coordinates (LC). (n) Schematic drawing of two domains in nematic state in lab coordinates. The two domains are rotated $90^{\circ}$ with each other. For domain $V$, the $x(y)$ axis is parallel to $X(Y)$ axis in LC while for domain H, it is reversed. (o) Summary of the allowed orbitals under LV and LH polarization geometries defined for lab coordinates and each domain along the high-symmetry cuts. (p)-(q) The photoemission spectra (EDCs) on the Fermi surface of two domains. The location of the 4-momenta is marked in (e). For each EDC, the peak area is obtained after subtracting the background. The obtained area ratio between the P1 and $\mathrm{P} 1^{\prime}$ points is 7.3 in (p) and between the $\mathrm{P} 2$ and $\mathrm{P} 2^{\prime}$ points is 5.3 in (q). The ratio is used to estimate the orbital components of $d_{x z}$ and $d_{y z}$ for the measured momentum points.

The polarization measurements on twinned samples also provide a unique way to determine the orbital character on the Fermi surface in a quantitative manner [16]. This is realized by comparing the measured spectral weight on the Fermi surface of one horizontal domain and its equivalent Fermi surface of another vertical domain. In Fig. 2(e), the measured Fermi surface mapping consists of signals from the horizontal domain (solid lines) and vertical domain (dashed lines), also as schematically shown in Fig. 2(k). P1 and $\mathrm{P} 2$ are the vertex points of the inner and outer Fermi surface for the horizontal domain while $\mathrm{P}^{\prime}{ }^{\prime}$ and $\mathrm{P} 2^{\prime}$ are the equivalent points on the Fermi surface of another vertical domain. Under the LV polarization [Fig. 2(e)], according to the matrix element analysis [Fig. 2(o)], only $d_{x z}$ orbital is allowed for the P1 and P2 points while the $d_{y z}$ orbital is fully forbidden for the horizontal domain. On the other hand, for $\mathrm{P}^{\prime}$ and $\mathrm{P} 2^{\prime}$ points for the vertical domain, the $d_{y z}$ orbital is allowed while the $d_{x z}$ orbital is forbidden under the same LV polarization geometry [Fig. 2(o)]. Therefore, the $d_{x z}$ and $d_{y z}$ orbital components for the given momentum point, $\mathrm{P} 1$ or $\mathrm{P} 2$, can be determined from the spectral weight at the two equivalent points from the two orthogonal twins [16]. Figure 2(p) shows the measured photoemission spectra [energy distribution curves (EDCs)] for P1 and $\mathrm{P}^{\prime}$ points marked in Fig. 2(e), and the EDCs for P2 and P2' points are shown in Fig. 2(q). From the EDC peak area, we estimated that for the P1 point, it consists of $\sim 88 \% d_{x z}$ orbital and $\sim 12 \% d_{y z}$ orbital, while for the P2 point it consists of $\sim 84 \% d_{x z}$ orbital and $\sim 16 \% d_{y z}$ orbital. These results confirm that the vertex area of the vertical long axis is dominated by the $d_{x z}$ orbital [Fig. 1(e)].

Figure 3 shows the band structure of FeSe measured along two high-symmetry directions under different polarization geometries. Different polarization measurements help provide a complete band structure because the band may show different intensities under different polarization geometries due to the photoemission matrix element effects. The polarization measurements can also help identify the orbital character of the measured bands. To see the measured bands more clearly, we also show the second derivative 

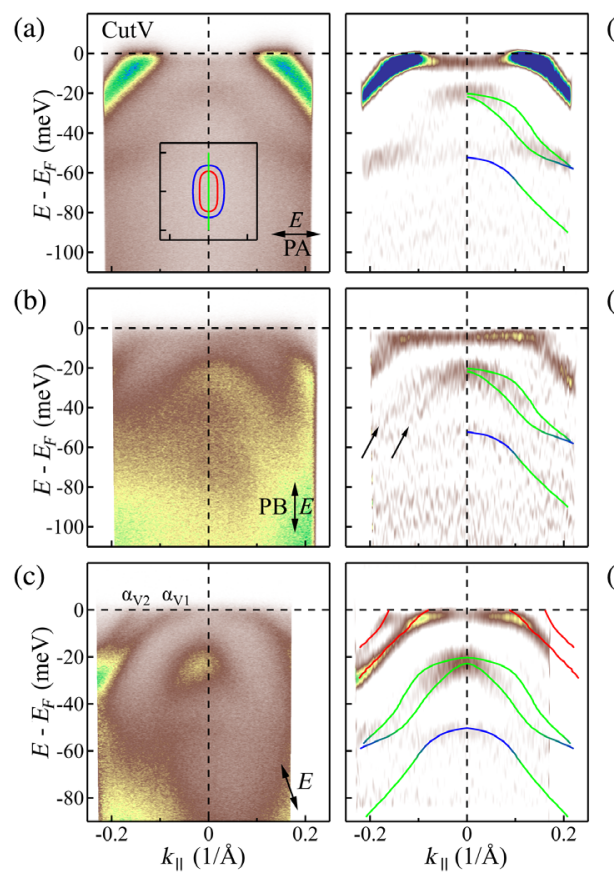

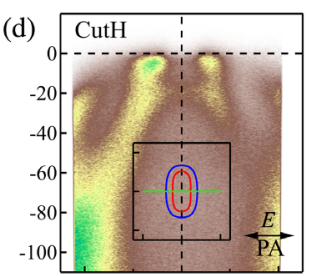

(e)

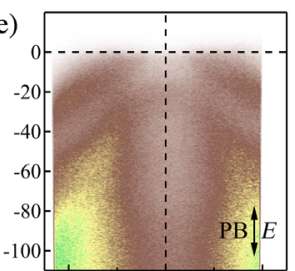

(f)

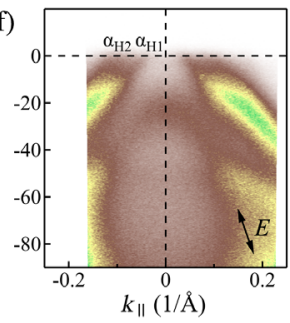

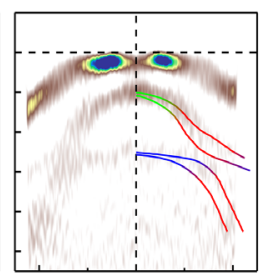
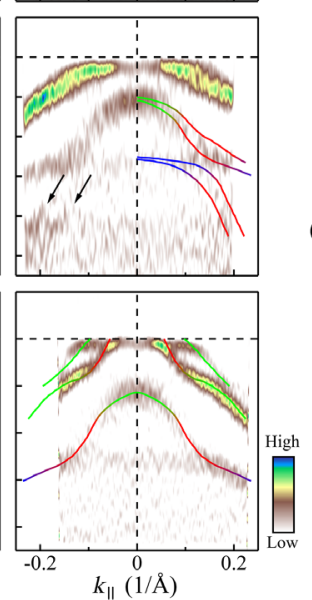
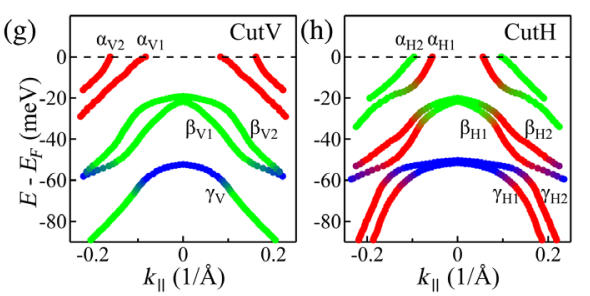

(i)

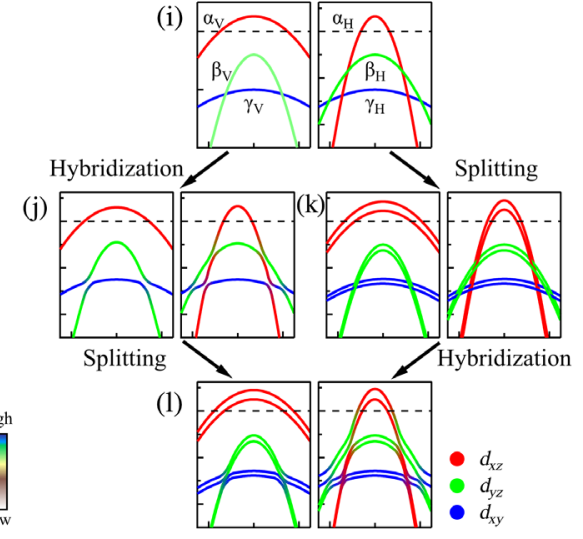

FIG. 3. Band structure of single-domain FeSe along high-symmetry cuts and their orbital nature. (a)-(c) Band structure measured along the vertical momentum cut under PA (a), PB (b), and a mixed (c) polarization geometries. The location of the momentum cut is shown in the inset of (a). The left-hand panels of (a)-(c) represent the original data while the right-hand panels are corresponding second derivative images with respect to energy in order to enhance the contrast. Some observed bands are marked by the guide lines and arrows. (d)-(f) Same as (a)-(c) but for the horizontal momentum cut as marked in the inset of (d). (g), (h) Extracted band structure for the vertical momentum cut (g) and horizontal momentum cut (h) obtained from (a)-(c) and (d)-(f), respectively. The observed bands are labeled as three branches of $\alpha, \beta$, and $\gamma$. (i) Schematic of the band structure along the vertical (left-hand panel) and horizontal (right-hand panel) momentum cuts from $\alpha$ (red lines), $\beta$ (green lines), and $\gamma$ (blue lines) orbitals. (j) Same as (i) but only considering the hybridization between different bands. (k) Same as (i) but only considering the splitting of each band. (l) Same as (i) but considering both band hybridization and splitting of each band. The red, green, and blue colors represent the $d_{x z}, d_{y z}$, and $d_{x y}$ orbitals, respectively.

images [right-hand panels in Figs. 3(a)-3(f)] to enhance the contrast, in addition to the original data [left-hand panels in Figs. 3(a)-3(f)]. Figures 3(g) and 3(h) summarize the observed bands along the vertical $k_{y}$ and the horizontal $k_{x}$ directions, obtained from the data in Figs. 3(a)-3(c) and Figs. 3(d)-3(f), respectively. Overall, in the measured energy range, three branches of bands [labeled as $\alpha, \beta$, and $\gamma$ in Figs. 3(g) and 3(h)] are observed for both the momentum cuts, and each branch may be further split into two subbranch bands. Similar to the double Fermi surface observation in Fig. 1, measurements using the mixed polarization geometry [Figs. 3(c) and 3(f)] are more desirable to observe clear band splittings, in particular, for the $\alpha$ band. For the bands of the vertical cut [Fig. 3(g)], the $\alpha_{V 1}$ and $\alpha_{V 2}$ bands are clearly observed in the raw data [Fig. 3(c)]; the $\beta_{V 1}$ and $\beta_{V 2}$ bands can be identified in the second derivative image [Fig. 3(b)] and the weak $\gamma_{V}$ band can be seen in all the second derivative images [Figs. 3(a)-3(c)]. For the bands of the horizontal cut [Fig. 3(h)], the $\alpha_{H 1}$ and $\alpha_{H 2}$ bands are clearly observed in the raw data [Fig. 3(f)]; the $\beta_{H 1}$ and $\beta_{H 2}$ bands can be identified in the second derivative image [Fig. 3(e)] and the weak $\gamma_{H 1}$ and $\gamma_{\mathrm{H} 2}$ bands can be seen more clearly in the second derivative image [Fig. 3(e)].
Our results provide direct evidence on the band splitting and the coexistence of two holelike Fermi pockets around the Brillouin zone center. We note that signatures of the two holelike Fermi pockets and the associated band splitting were present in previous measurements although only one Fermi surface was considered [10,14,19,20,23,24,50]. In previous ARPES measurements, at least three bands are clearly resolved crossing the Fermi level at photon energies between 25 and $30 \mathrm{eV}$ along a momentum cut crossing the $\Gamma$ point [Fig. S3 in Supplemental Material [49]) [10]. This cannot be explained by a single hole-pocket picture which will give at most two bands because of two coexisting orthogonal domains; instead, it is consistent with the two hole-pocket scenario we discovered in the present work. In previous STM measurements, two holelike bands are clearly identified along the momentum cut crossing the $\Gamma$ point in a single-domain FeSe. This was interpreted in terms of single hole-pocket picture, and two bands were assigned to bands at different $k_{z}$ 's [20]. With our discovery of two hole pockets around the zone center, the STM observation of two holelike bands would have an alternative and natural explanation. In quantum oscillation measurements of $\mathrm{FeSe}$, the number of oscillation frequencies observed is controversial 
that varies from three [24] to four [10] to six [23]. The assignment of these oscillations to the Fermi surface is also under debate $[10,23,24]$. Our direct ARPES observation of double holelike Fermi surface sheets around the zone center is important for the quantum oscillation measurements to reexamine their results and make a more consistent assignment.

Now we discuss the origin of the band splitting and double Fermi pockets near $\Gamma$ observed in FeSe. We first examine whether these observations can be attributed to the $k_{z}$ effect. In the photoemission process, the relatively short photoelectron escape depth gives rise to a finite momentum resolution of $k_{z}$, and the measured signal can then be a summation over a finite range of $\Delta k_{z}$ [51]. As mentioned above, in FeSe, at least three bands can be clearly observed around the zone center at some photon energies between 25 and $30 \mathrm{eV}$ (see Fig. S3 in Supplemental Material [49]) [10]. Each of these multiple bands exhibits obvious $k_{z}$ dispersion between 20 and $50 \mathrm{eV}$ photon energy (Fig. S3 in Supplemental Material [49]). This indicates that, even for the photon energy of $20-50 \mathrm{eV}$, the $k_{z}$ resolution is good enough to measure the band dispersion for a particular $k_{z}$ value, definitely not the summation over the $k_{z}$ range between 0 and 1. The $6.994 \mathrm{eV}$ laser we used corresponds to a $k_{z}$ of $0.47 \pi / c$ that lies nearly in the middle of the basal $k_{z}=0$ and $k_{z}=1$ planes [10] that has a similar $k_{z}$ value as that of $29 \mathrm{eV}$ photon energy (Fig. S3 in Supplemental Material [49]). It is expected that the $6.994 \mathrm{eV}$ laser we used has an enhanced bulk sensitivity and better $k_{z}$ momentum resolution than higher photon energies [52]. Therefore, our observation of two separate Fermi pockets cannot be due to finite $k_{z}$ resolution because the summation over a large range of $k_{z}$ between 0 and 1 would give a continuous spectrum rather than two discrete features we observed. Instead, the observed double Fermi pockets represent the intrinsic band structure for the particular $k_{z}=0.47 \pi / c$, consistent with the observation of at least three bands at the equivalent $29 \mathrm{eV}$ photon energy in FeSe with two domains (Fig. S3 in Supplemental Material [49]). The clear $k_{z}$ dispersion of these multiple bands also indicates that they are bulk electronic states, rather than the surface states.

Three branches of bands have been observed in FeSe around the zone center by previous ARPES measurements [5-19]. The polarization measurements combined with the band structure calculations have indicated that the observed three branches of bands are mainly derived from the $d_{x z}$, $d_{y z}$, and $d_{x y}$ orbitals, as schematically shown in Fig. 3(i) when the nematicity is included without considering the hybridization between bands. From the measured band structure in Figs. $3(\mathrm{~g})$ and $3(\mathrm{~h})$, it is clear that band hybridization occurs at all the band crossing points in Fig. 3(j). The resultant band structure by considering these band hybridizations in Fig. 3(j) shows a good agreement with the measured results [Figs. 3(g) and 3(h)] without considering the band splitting. However, even though the nematicity, band hybridization, and spin-orbit coupling are all considered, only three bands in the measured energy range are expected [Figs. 3(i) and 3(j)]. It is impossible to explain our observation of up to six bands in Figs. 3(g) and 3(h). Only when a new mechanism is invoked that can cause further band splitting of the three bands $(\alpha, \beta$, and $\gamma)$ can we get a band picture [Fig. 3(1)] that is possibly consistent with the measured results [Figs. 3(g) and 3(h)].

The observations of double Fermi pockets and band splitting in FeSe indicate that an additional mechanism is at play in addition to the spin-orbit coupling and the nematic order. The spin-orbit coupling can only lift the degeneracy of $d_{x z} / d_{y z}$ bands at the $\mathrm{BZ}$ center. The fourfold rotational symmetry breaking from the nematicity can further enhance the splitting between the $d_{x z}$ and $d_{y z}$ bands. But neither of them can cause the splitting of the $d_{x z}$ or $d_{y z}$ band. Therefore, there should be an additional symmetry breaking that occurs in the nematic phase. We have considered two possible candidates to carry out theoretical simulations (see Methods below) and compare them with our measurements. One is the time-reversal symmetry breaking (ferromagnetic order) and the other is the inversion symmetry breaking (Rashba spin-orbit coupling), which may originate from the asymmetrical orbital order on the top and bottom Se sites or two Fe sites. Figure 4 shows the measured Fermi surface and the detailed momentum dependence of band structure for FeSe measured under different polarization geometries. The calculated Fermi surface and band structure are shown in Figs. 4(g) and 4(h) and Figs. 4(i) and 4(j) for the ferromagnetic ordering and the Rashba spin splitting effects, respectively. In the case with a ferromagnetic order [Fig. 4(i)], the prominent features are that the $d_{x z}, d_{y z}$, and $d_{x y}$ bands are split and the splitting for the $d_{x z} / d_{y z}$ bands reaches the maximum at the $\Gamma$ point and decreases away from the $\Gamma$ point. In the latter case, the inversion symmetry breaking introduces Rashba spin-orbit coupling. In contrast to the ferromagnetic ordering, the splitting for the $d_{x z} / d_{y z}$ bands vanishes at the $\Gamma$ point and increases away from the $\Gamma$ point. It can be found that in terms of current experimental precision, both theoretical calculation results capture well our ARPES measurement results from the Fermi surface topology, the orbital nature evolution, and band dispersions along the Fermi surface angles $\theta$. In particular, the double Fermi surface around $\Gamma$ and their orbital nature are both consistent with our experimental results shown in Fig. 1(e).

The observed two pockets and band splitting in the nematic phase of FeSe indicate there is an additional order that breaks either the time-reversal symmetry or the inversion symmetry. They provide important implications in understanding the superconducting pairing in FeSe. For the ferromagnetic ordering scenario, as each pocket has the same spin, the spin triplet $p$-wave pairing state is likely to be dominant, which is consistent with the 

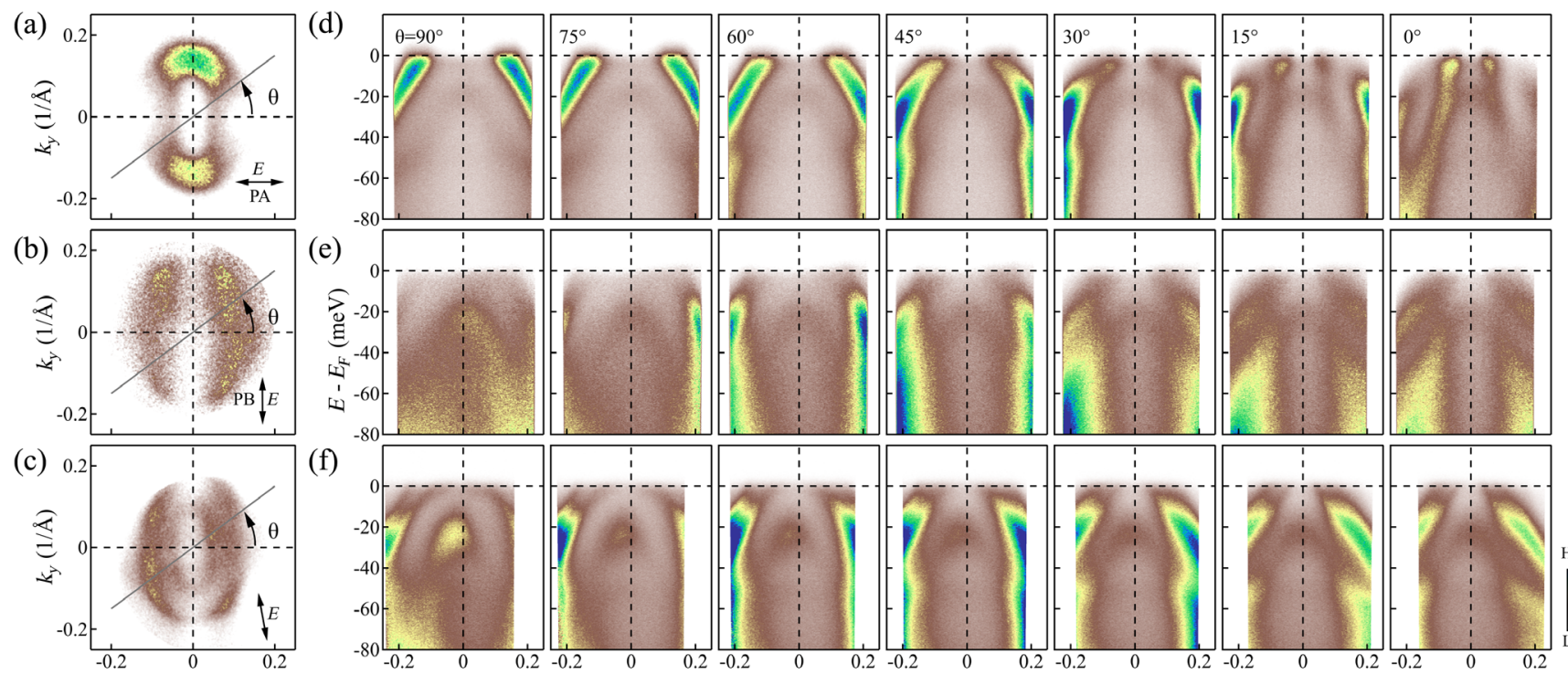

(f)
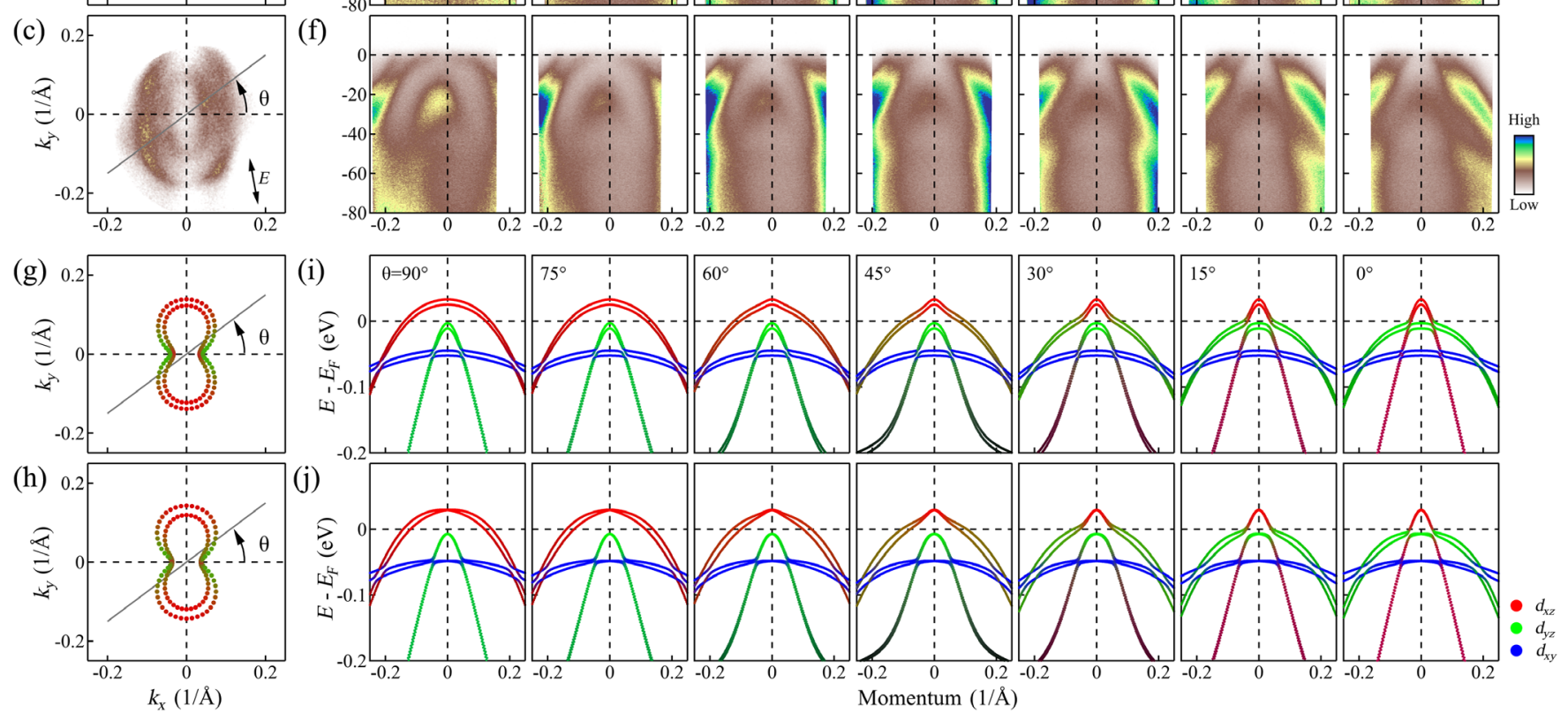

FIG. 4. Calculated Fermi surface and band structure of FeSe and their comparison with the measured results. (a)-(c) The Fermi surface mappings of the single-domain FeSe measured under the PA (a), the PB (b), and a mixed (c) polarization geometries. The momentum cuts here are defined by the Fermi surface angle $\theta$. (d)-(f) Band structure for the momentum cuts at different Fermi surface angles $\theta$ corresponding to (a)-(c). (g),(h) Calculated Fermi surface around $\Gamma$ point in considering the ferromagnetic order and the Rashba spinorbit coupling in the nematic state of FeSe, respectively. Their corresponding band structures are shown in (i),(j). The $d_{x z}, d_{y z}$, and $d_{x y}$ orbital components are represented by red, green, and blue colors, respectively.

measured gap function $[14,15]$. Signatures of the timereversal symmetry breaking were reported at the twin boundaries of FeSe in the superconducting state [53]. For the Rashba scenario, due to the inversion symmetry breaking, spin singlet and spin triplet pairing in principle can mix and an exotic pairing state may occur. Moreover, the two pockets have the opposite spin texture, a sign change between the gap functions on those pockets can render FeSe in a topologically nontrivial phase [54], which can host Majorana edge modes. Further measurements are needed to distinguish these two scenarios; in particular, spin resolved experiments can provide a verdict because the Fermi surfaces show distinct spin textures between these two cases.

In summary, by carrying out high-resolution laser-based ARPES measurements on FeSe, double holelike Fermi surface and band splitting are clearly observed for the first time around the BZ center in the nematic phase of FeSe.
The new Fermi surface topology asks for reexamination of the previous theoretical and experimental understanding of FeSe. These results indicate that, in addition to the nematic order and spin-orbit coupling, there is an extra hidden order in FeSe that breaks either inversion or time-reversal symmetries. To the best of our knowledge, no candidate of such a new order has been identified experimentally. We hope our present work can stimulate further effort to identify the hidden order and provide new information in understanding the nematicity and superconductivity in FeSe.

Methods: Sample.-High-quality FeSe single crystals were grown by $\mathrm{KCl} / \mathrm{AlCl}_{3}$ chemical vapor transport technique [55]. The samples measured here have a $T_{c}$ of $9.2 \mathrm{~K}$ with a transition width of $\sim 0.2 \mathrm{~K}$.

ARPES measurements.-High-resolution ARPES measurements were performed at our newly developed laserbased system equipped with the $6.994 \mathrm{eV}$ vacuum ultraviolet 
laser and the time-of-flight electron energy analyzer (ARTOF $10 \mathrm{k}$ by Scienta Omicron) $[15,48,56]$. This latest-generation ARPES system is capable of measuring photoelectrons covering two-dimensional momentum space $\left(k_{x}, k_{y}\right)$ simultaneously. The system is equipped with an ultralow-temperature cryostat which can cool the sample to a low temperature of $1.6 \mathrm{~K}$. The ARPES measurements were carried out using various polarizations of the laser light. The laser spot size on the sample is about $70 \mu \mathrm{m}$. The overall energy resolution combining the laser linewidth and the ARTOF electron energy analyzer is $\sim 1 \mathrm{meV}$. The angular resolution of the analyzer is $\sim 0.1^{\circ}$, corresponding momentum resolution of $0.0015 \AA^{-1}$ at a photon energy of $6.994 \mathrm{eV}$. The Fermi level is referenced by measuring polycrystalline gold, which is in good electrical contact with the sample, as well as the normal state measurement of the sample above $T_{c}$. The samples were cleaved in situ and measured at different temperatures in ultrahigh vacuum with a base pressure better than $5.0 \times 10^{-11}$ mbar.

Electronic structure simulations.-To simulate the band structure of $\mathrm{FeSe}$, we adopted a five-orbital tight-binding model [57-59] including on-site spin-orbit coupling $(\lambda)$. In the nematic state, to explain the observed band splitting around the $\Gamma$ point, we further consider an $s$-wave nematic order $\left(\Delta_{s}\right)$ which lifts the degeneracy of $d_{x z} / d_{y z}$ bands and breaks the $C_{4}$ rotational symmetry. The Hamiltonian of $s$-wave orders is given by

$$
H_{s}=\sum_{\boldsymbol{k}} \Delta_{s}\left(\cos k_{x}+\cos k_{y}\right)\left(n_{x z, \boldsymbol{k}}-n_{y z, \boldsymbol{k}}\right),
$$

where $n_{\alpha, \boldsymbol{k}}=n_{\alpha, \boldsymbol{k} \uparrow}+n_{\alpha, \boldsymbol{k} \downarrow}$ is the density for $\alpha$ orbital. To model the observed band splitting in ARPES experiments, we consider two possible origins: ferromagnetic ordering and Rashba spin-orbit coupling. The ferromagnetic Hamiltonian term is given by

$$
H_{\mathrm{FM}}=\sum_{\boldsymbol{k} \alpha \sigma_{1} \sigma_{2}} \boldsymbol{M} \cdot \boldsymbol{\sigma}_{\sigma_{1} \sigma_{2}} c_{\alpha k \sigma_{1}}^{\dagger} c_{\alpha k \sigma_{2}},
$$

where $\boldsymbol{M}$ is the total magnetization. If the inversion symmetry is broken in the nematic phase, Rashba spinorbit coupling can occur and the term for $d_{x z}$ and $d_{y z}$ orbitals reads

$$
\begin{aligned}
H_{R}= & \sum_{\boldsymbol{k}}\left[-2\left(i \lambda_{1} \sin k_{x}+\lambda_{2} \sin k_{y}\right) c_{x z, \boldsymbol{k} \uparrow}^{\dagger} c_{x z, \boldsymbol{k} \downarrow}\right. \\
& -2\left(i \lambda_{2} \sin k_{x}+\lambda_{1} \sin k_{y}\right) c_{y z, \boldsymbol{k} \uparrow}^{\dagger} c_{y z, \boldsymbol{k} \downarrow} \\
& \left.-2 \lambda_{3}\left(i \sin k_{x}+\sin k_{y}\right) c_{x y, \boldsymbol{k} \uparrow}^{\dagger} c_{x y, \boldsymbol{k} \downarrow}+\text { H.c. }\right] .
\end{aligned}
$$

In the nematic phase, the adopted parameters in the main text are $\lambda=10 \mathrm{meV}$ and $\Delta_{s}=8.75 \mathrm{meV}$. For the scenario of ferromagnetic order induced splitting, the magnetization is assumed along the $z$ direction and $M_{z}=4 \mathrm{meV}$ is adopted. For the scenario of Rashba spin-orbit coupling induced splitting, $\lambda_{1}=\lambda_{2}=10 \mathrm{meV}$ and $\lambda_{3}=5 \mathrm{meV}$ are used.

We are thankful for financial support from the National Key Research and Development Program of China (Grants No. 2016YFA0300300, No. 2017YFA0302900, No. 2018YFA0704200, and No. 2019YFA0308000), the National Natural Science Foundation of China (Grants No. 11888101, No. 11874405), the Strategic Priority Research Program (B) of the Chinese Academy of Sciences (Grant No. XDB25000000), the Youth Innovation Promotion Association of CAS (Grant No. 2017013), and the Research Program of Beijing Academy of Quantum Information Sciences (Grant No. Y18G06).

C. L., X.X.W., and L.W. contributed equally to this work. X. J.Z., C. L., and L. Z. proposed and designed the research. L. W. and Y. G.S. contributed to FeSe crystal growth. X. X. W., C. X. D., J. P. H., and T. X. contributed to the band structure calculations and theoretical discussion. C. L., D. F. L., Y. Q. C., Y.W., Q. G., C. Y.S., J. W. H., J. L., P. A., G. D. L., Y. H., Q. Y. W., F. F. Z., S. J.Z., F. Y., Z. M. W., Q. J.P., Z. Y.X., L.Z., and X. J.Z. contributed to the development and maintenance of Laser-ARTOF system. C. L., Q. G., and X. W. J. contributed to software development for data analysis. C. L., Y.Q.C., and Y.W. carried out the experiment with assistance from Q. G., C. Y. S., J. W. H., J. L., P. A., H. L. L., and C. H. Y.. C. L., D. F. L., L. Z., and X. J.Z. analyzed the data. C. L., L.Z., and X.J.Z. wrote the paper with X.X. W., J.P.H., and T. X. All authors participated in discussion and comment on the paper.

[1] F. Hsu et al., Superconductivity in the PbO-type Structure $\alpha$-FeSe, Proc. Natl. Acad. Sci. U.S.A. 105, 14262 (2008).

[2] T. M. McQueen, A. J. Williams, P. W. Stephens, J. Tao, Y. Zhu, V. Ksenofontov, F. Casper, C. Felser, and R. J. Cava, Tetragonal-to-Orthorhombic Structural Phase Transition at $90 \mathrm{~K}$ in the Superconductor $\mathrm{Fe}_{1.01} \mathrm{Se}$, Phys. Rev. Lett. 103, 057002 (2009).

[3] S. Medvedev et al., Electronic and Magnetic Phase Diagram of $\beta-\mathrm{Fe}_{1.01}$ Se with Superconductivity at $36.7 \mathrm{~K}$ Under Pressure, Nat. Mater. 8, 630 (2009).

[4] J. P. Sun et al., Dome-Shaped Magnetic Order Competing with High-Temperature Superconductivity at High Pressures in FeSe, Nat. Commun. 7, 12146 (2016).

[5] J. Maletz, V. B. Zabolotnyy, D. V. Evtushinsky, S. Thirupathaiah, A. U. B. Wolter, L. Harnagea, A. N. Yaresko, A. N. Vasiliev, D. A. Chareev, A. E. Bohmer et al., Unusual Band Renormalization in the Simplest Iron-Based Superconductor $\mathrm{FeSe}_{1-x}$, Phys. Rev. B 89, 220506(R) (2014).

[6] K. Nakayama, Y. Miyata, G. N. Phan, T. Sato, Y. Tanabe, T. Urata, K. Tanigaki, and T. Takahashi, Reconstruction of 
Band Structure Induced by Electronic Nematicity in an FeSe Superconductor, Phys. Rev. Lett. 113, 237001 (2014).

[7] T. Shimojima et al., Lifting of $x z / y z$ Orbital Degeneracy at the Structural Transition in Detwinned FeSe, Phys. Rev. B 90, 121111(R) (2014).

[8] P. Zhang et al., Observation of Two Distinct $d_{x z} / d_{y z}$ Band Splittings in FeSe, Phys. Rev. B 91, 214503 (2015).

[9] Y. Suzuki, T. Shimojima, T. Sonobe, A. Nakamura, M. Sakano, H. Tsuji, J. Omachi, K. Yoshioka, M. KuwataGonokami, T. Watashige et al., Momentum-Dependent Sign Inversion of Orbital Order in Superconducting FeSe, Phys. Rev. B 92, 205117 (2015).

[10] M. D. Watson et al., Emergence of the Nematic Electronic State in FeSe, Phys. Rev. B 91, 155106 (2015).

[11] M. D. Watson, T. K. Kim, L. C. Rhodes, M. Eschrig, M. Hoesch, A. A. Haghighirad, and A. I. Coldea, Evidence for Unidirectional Nematic Bond Ordering in FeSe, Phys. Rev. B 94, 201107(R) (2016).

[12] M. D. Watson, A. A. Haghighirad, L. C. Rhodes, M. Hoesch, and T. K. Kim, Electronic Anisotropies Revealed by Detwinned Angle-Resolved Photoemission Spectroscopy Measurements of FeSe, New J. Phys. 19, 103021 (2017).

[13] H. C. Xu, X. H. Niu, D. F. Xu, J. Jiang, Q. Yao, Q. Y. Chen, Q. Song, M. Abdel-Hafiez, D. A. Chareev, A. N. Vasiliev et al., Highly Anisotropic and Twofold Symmetric Superconducting Gap in Nematically Ordered $\mathrm{FeSe}_{0.93} \mathrm{~S}_{0.07}$, Phys. Rev. Lett. 117, 157003 (2016).

[14] T. Hashimoto et al., Superconducting Gap Anisotropy Sensitive to Nematic Domains in FeSe, Nat. Commun. 9, 282 (2018).

[15] D. Liu, C. Li, J. Huang, B. Lei, L. Wang, X. Wu, B. Shen, Q. Gao, Y. Zhang, X. Liu et al., Orbital Origin of Extremely Anisotropic Superconducting Gap in Nematic Phase of FeSe Superconductor, Phys. Rev. X 8, 031033 (2018).

[16] L. C. Rhodes, M. D. Watson, A. A. Haghighirad, D. V. Evtushinsky, M. Eschrig, and T. K. Kim, Scaling of the Superconducting Gap with Orbital Character in FeSe, Phys. Rev. B 98, 180503(R) (2018).

[17] Y. Kushnirenko, A. V. Fedorov, E. Haubold, S. Thirupathaiah, T. Wolf, S. Aswartham, I. Morozov, T. K. Kim, B. Büchner, and S. V. Borisenko, Three-Dimensional Superconducting Gap in FeSe from Angle-Resolved Photoemission Spectroscopy, Phys. Rev. B 97, 180501(R) (2018).

[18] H. Pfau et al., Momentum Dependence of the Nematic Order Parameter in Iron-Based Superconductors, Phys. Rev. Lett. 123, 066402 (2019).

[19] M. D. Watson, A. A. Haghighirad, H. Takita, W. Mansuer, H. Iwasawa, E. F. Schwier, A. Ino, and M. Hoesch, Shifts and Splittings of the Hole Bands in the Nematic Phase of FeSe, J. Phys. Soc. Jpn. 86, 053703 (2017).

[20] S. Kasahara et al., Field-Induced Superconducting Phase of $\mathrm{FeSe}$ in the BCS-BEC Crossover, Proc. Natl. Acad. Sci. U.S.A. 111, 16309 (2014).

[21] P. Sprau, A. Kostin, A. Kreisel, A. E. Böhmer, V. Taufour, P. C. Canfield, S. Mukherjee, P. J. Hirschfeld, B. M. Andersen, and J. C. S. Davis, Discovery of Orbital-Selective Cooper Pairing in FeSe, Science 357, 75 (2017).

[22] T. Hanaguri, S. Kasahara, J. Boker, I. Eremin, T. Shibauchi, and Y. Matsuda, Quantum Vortex Core and Missing
Pseudogap in the Multiband BCS-BEC Crossover Superconductor FeSe, Phys. Rev. Lett. 122, 077001 (2019).

[23] T. Terashima, N. Kikugawa, A. Kiswandhi, E. S. Choi, J. S. Brooks, S. Kasahara, T. Watashige, H. Ikeda, T. Shibauchi, Y. Matsuda et al., Anomalous Fermi Surface in FeSe Seen by Shubnikov-de Haas Oscillation Measurements, Phys. Rev. B 90, 144517 (2014).

[24] M. D. Watson et al., Dichotomy between the Hole and Electron Behavior in Multiband Superconductor FeSe Probed by Ultrahigh Magnetic Fields, Phys. Rev. Lett. 115, 027006 (2015).

[25] J. Guo, S. Jin, G. Wang, S. Wang, K. Zhu, T. Zhou, M. He, and $\mathrm{X}$. Chen, Superconductivity in the Iron Selenide $\mathrm{K}_{x} \mathrm{Fe}_{2} \mathrm{Se}_{2}(0 \leq x \leq 1)$, Phys. Rev. B 82, 180520(R) (2010).

[26] T. P. Ying, X. Chen, G. Wang, S. Jin, X. Lai, T. Zhou, H. Zhang, S. Shen, and W. Wang, Superconducting Phases in Potassium-Intercalated Iron Selenides, J. Am. Chem. Soc. 135, 2951 (2013).

[27] T. P. Ying, X. L. Chen, G. Wang, S. F. Jin, T. T. Zhou, X. F. Lai, H. Zhang, and W. Y. Wang, Observation of Superconductivity at 30-46 $\mathrm{K}$ in $\mathrm{A}_{x} \mathrm{Fe}_{2} \mathrm{Se}_{2}(\mathrm{~A}=\mathrm{Li}, \mathrm{Na}, \mathrm{Ba}, \mathrm{Sr}$, $\mathrm{Ca}, \mathrm{Yb}$, and Eu), Sci. Rep. 2, 426 (2012).

[28] A. K. Maziopa, E. V. Pomjakushina, V. Yu. Pomjakushin, F. von Rohr, A. Schilling, and K. Conder, Synthesis of a New Alkali Metal-Organic Solvent Intercalated Iron Selenide Superconductor with $T_{c} \sim 45 \mathrm{~K}$, J. Phys. Condens. Matter 24, 382202 (2012).

[29] M. B. Lucas et al., Enhancement of the Superconducting Transition Temperature of FeSe by Intercalation of a Molecular Spacer Layer, Nat. Mater. 12, 15 (2013).

[30] T. Hatakeda, T. Noji, T. Kawamata, M. Kato, and Y. Koike, New Li-Ethylenediamine-Intercalated Superconductor $\mathrm{Li}_{x}\left(\mathrm{C}_{2} \mathrm{H}_{8} \mathrm{~N}_{2}\right)_{y} \mathrm{Fe}_{2-z} \mathrm{Se}_{2}$ with $T_{c}=45 \mathrm{~K}$, J. Phys. Soc. Jpn. 82, 123705 (2013).

[31] M. Z. Shi, N. Z. Wang, B. Lei, C. Shang, F. B. Meng, L. K. Ma, F. X. Zhang, D. Z. Kuang, and X. H. Chen, OrganicIon-Intercalated FeSe-Based Superconductors, Phys. Rev. Mater. 2, 074801 (2018).

[32] X.F. Lu et al., Coexistence of Superconductivity and Antiferromagnetism in $\left(\mathrm{Li}_{0.8} \mathrm{Fe}_{0.2}\right) \mathrm{OHFeSe}$, Nat. Mater. 14, 325 (2015).

[33] B. Lei, J. H. Cui, Z. J. Xiang, C. Shang, N. Z. Wang, G. J. Ye, X. G. Luo, T. Wu, Z. Sun, and X. H. Chen, Evolution of High-Temperature Superconductivity from Low- $T_{c}$ Phase Tuned by Carrier Concentration in FeSe Thin Flakes, Phys. Rev. Lett. 116, 077002 (2016).

[34] B. Lei et al., Tuning Phase Transitions in FeSe Thin Flakes by Field-Effect Transistor with Solid Ion Conductor as the Gate Dielectric, Phys. Rev. B 95, 020503(R) (2017).

[35] J. Seo, B. Y. Kim, B. S. Kim, J. K. Jeong, J. M. Ok, J. S. Kim, J. D. Denlinger, S.-K. Mo, C. Kim, and Y. K. Kim, Superconductivity Below $20 \mathrm{~K}$ in Heavily Electron-Doped Surface Layer of FeSe Bulk Crystal, Nat. Commun. 7, 11116 (2016).

[36] Q. Y. Wang et al., Interface-Induced High-Temperature Superconductivity in Single Unit-Cell FeSe Films on $\mathrm{SrTiO}_{3}$, Chin. Phys. Lett. 29, 037402 (2012).

[37] D. F. Liu et al., Electronic Origin of High-Temperature Superconductivity in Single-Layer FeSe Superconductor, Nat. Commun. 3, 931 (2012). 
[38] S. L. He et al., Phase Diagram and Electronic Indication of High-Temperature Superconductivity at $65 \mathrm{~K}$ in SingleLayer FeSe Films, Nat. Mater. 12, 605 (2013).

[39] S. Y. Tan et al., Interface-Induced Superconductivity and Strain-Dependent Spin Density Waves in $\mathrm{FeSe} / \mathrm{SrTiO}_{3}$ Thin Films,. Nat. Mater. 12, 634 (2013).

[40] J. J. Lee et al., Interfacial Mode Coupling as the Origin of the Enhancement of $T_{c}$ in FeSe Films on $\mathrm{SrTiO}_{3}$, Nature (London) 515, 245 (2014).

[41] H. Hu, R. Yu, E. M. Nica, J. X. Zhu, and Q. Si, OrbitalSelective Superconductivity in the Nematic Phase of FeSe, Phys. Rev. B 98, 220503(R) (2018).

[42] J. Kang, R. M. Fernandes, and A. Chubukov, Superconductivity in FeSe: The Role of Nematic Order, Phys. Rev. Lett. 120, 267001 (2018).

[43] G. Y. Chen, X. Zhu, H. Yang, and H. H. Wen, Highly Anisotropic Superconducting Gaps and Possible Evidence of Antiferromagnetic Order in FeSe Single Crystals, Phys. Rev. B 96, 064524 (2017).

[44] P. K. Biswas, A. Kreisel, Q. Wang, D. T. Adroja, A. D. Hillier, J. Zhao, R. Khasanov, J.-C. Orain, A. Amato, and E. Morenzoni, Evidence of Nodal Gap Structure in the Basal Plane of the FeSe Superconductor, Phys. Rev. B 98, 180501(R) (2018).

[45] F. Hardy et al., Calorimetric Evidence of Nodal Gaps in the Nematic Superconductor FeSe, Phys. Rev. B 99, 035157 (2019).

[46] A. Kreisel, B. M. Andersen, P. O. Sprau, A. Kostin, J. C. S. Davis, and P. J. Hirschfeld, Orbital Selective Pairing and Gap Structures of Iron-Based Superconductors, Phys. Rev. B 95, 174504 (2017).

[47] L. Benfatto, B. Valenzuela, and L. Fanfarillo, Nematic Pairing from Orbital-Selective Spin Fluctuations in FeSe, npj Quantum Mater. 3, 56 (2018).

[48] X. J. Zhou, S. He, G. Liu, L. Zhao, L. Yu, and W. Zhang, New Developments in Laser-Based Photoemission Spectroscopy and Its Scientific Applications: A Key Issues Review, Rep. Prog. Phys. 81, 062101 (2018).

[49] See Supplemental Material at http://link.aps.org/ supplemental/10.1103/PhysRevX.10.031033 for details on electronic structure analysis of single domain FeSe, temperature dependent Fermi surface mappings of single domain $\mathrm{FeSe}$, and $\mathrm{kz}$ dispersion around the Brillouin zone center using different photon energies.

[50] L. Fanfarillo, J. Mansart, P. Toulemonde, H. Cercellier, P. LeFevre, F. Bertran, B. Valenzuela, L. Benfatto, and V. Brouet, Orbital-Dependent Fermi Surface Shrinking as a Fingerprint of Nematicity in FeSe, Phys. Rev. B 94, 155138 (2016).

[51] S. Hufner, in Photoelectron Spectroscopy: Principles and Applications, Springer Series in Solid State Sciences Vol. 82 (Springer, Berlin, 1995).

[52] G. D. Liu et al., Development of a Vacuum Ultra-Violet Laser-Based Angle-Resolved Photoemission System with a Super-High Energy Resolution Better than $1 \mathrm{meV}$, Rev. Sci. Instrum. 79, 023105 (2008).

[53] T. Watashige et al., Evidence for Time-Reversal Symmetry Breaking of the Superconducting State Near Twin-Boundary Interfaces in FeSe Revealed by Scanning Tunneling Spectroscopy, Phys. Rev. X 5, 031022 (2015).

[54] X. L. Qi, T. L. Hughes, and S. C. Zhang, Topological Invariants for the Fermi Surface of a Time-ReversalInvariant Superconductor, Phys. Rev. B 81, 134508 (2010).

[55] A. E. Böhmer, F. Hardy, F. Eilers, D. Ernst, P. Adelmann, P. Schweiss, T. Wolf, and C. Meingast, Lack of Coupling between Superconductivity and Orthorhombic Distortion in Stoichiometric Single-Crystalline FeSe, Phys. Rev. B 87, 180505(R) (2013).

[56] Y. Zhang et al., Electronic Evidence of Temperature Induced Lifshitz Transition and Topological Nature in $\mathrm{ZrTe}_{5}$, Nat. Commun. 8, 15512 (2017).

[57] Y. Su, H. Liao, and T. Li, The Form and Origin of Orbital Ordering in the Electronic Nematic Phase of Iron-Based Superconductors, J. Phys. Condens. Matter 27, 105702 (2015).

[58] X. Wu et al., Nematic Orders and Nematicity-Driven Topological Phase Transition in FeSe, arXiv:1603.02055.

[59] T. Li and Y. Su, Driving Force of the Orbital-Relevant Electronic Nematicity in Fe-Based Superconductors, J. Phys. Condens. Matter 29, 425603 (2017). 